\title{
Formaldeído e acetaldeído atmosféricos no campus da Universidade de Hiroshima, Japão
}

\section{Atmospheric formaldehyde and acetaldehyde at the campus University of Hiroshima, Japan}

\author{
Sonia Naomi Nomi ${ }^{1}$; Hiroshi Sakugawa ${ }^{2}$, Kazuhiko Takeda ${ }^{2}$; Maria Cristina \\ Solci $^{1}$
}

Resumo

Formaldeído ( $\mathrm{HCHO}$ ) e acetaldeído $\left(\mathrm{CH}_{3} \mathrm{CHO}\right)$ atmosféricos foram determinados em Hiroshima (Japão) na primavera e verão em 2005. Simultaneamente foram determinadas as concentrações de ozônio $\left(\mathrm{O}_{3}\right)$, óxidos de nitrogênio $\left(\mathrm{NO}\right.$ e $\left.\mathrm{NO}_{2}\right)$ e dióxido de enxofre $\left(\mathrm{SO}_{2}\right)$ para obtenção do perfil temporal e das fontes dos aldeídos. Os aldeídos foram coletados utilizando cartuchos $\mathrm{C} 18$ revestidos com solução ácida de 2,4-DNPH e analisados por cromatografia líquida de alta eficiência com detecção UV/Vis em 360 $\mathrm{nm}$. As concentrações dos aldeídos foram mais elevadas no verão do que na primavera. Na primavera os intervalos de concentração de formaldeído (FA) e acetaldeído (AA) foram de 0,50 a 1,05 ppb e de 0,17 a 1,31 ppb e no verão os intervalos de concentração foram de 1,55 a 4,12 ppb e de 0,34 a 2,03 ppb, respectivamente. A média das razões FA/AA na primavera e no verão foram 3,09 e 3,72 sugerindo como fontes as emissões biogênicas dos aldeídos. Os níveis de concentração de FA e AA, a variação temporal e as razões FA/AA indicaram que as reações fotoquímicas contribuíram significativamente para a formação destas espécies no local de estudo.

Palavras-chave: Aldeídos. Razão FA/AA. Emissões biogênicas.

\begin{abstract}
Ambient levels of formaldehyde ( $\mathrm{HCHO})$ and acetaldehyde $\left(\mathrm{CH}_{3} \mathrm{CHO}\right)$ were measured at Hiroshima (Japan) during spring and summer of 2005. Measurements of $\mathrm{O}_{3}, \mathrm{NO}_{x}$, and $\mathrm{SO}_{2}$ were simultaneously conducted, in an attempt to identify temporal profiles and sources for these aldehydes. Atmospheric aldehydes were collected using $\mathrm{C}_{18}$ silica gel cartridges coated with an acidic solution of 2, 4-dinitrophenylhydrazine (2,4-DNPH) and analyzed by high performance liquid chromatography with UV/VIS detection adjusted to $360 \mathrm{~nm}$. Summer concentrations were higher than spring levels. FA concentrations during spring period ranged from 0.50 to $1.05 \mathrm{ppb}$ and AA concentrations ranged from 0.17 to $1.31 \mathrm{ppb}$; during summer their concentrations ranged from 1.55 to $4.12 \mathrm{ppb}$ and 0.34 to $2.03 \mathrm{ppb}$. Average FA/AA in spring and summer were 3.09 and 3.72 suggesting biogenic emissions of aldehydes. The levels of FA and AA, their temporal variations, and their concentration ratio (FA/ AA) indicated that photochemical reactions contributed significantly to the formation of atmospheric aldehydes at the study site.

Key-words: Aldehyde. FA/AA ratios. Biogenic emissions.
\end{abstract}

${ }^{1}$ Graduada e Mestre em Química dos Recursos Naturais, Universidade Estadual de Londrina.

${ }^{2}$ Docentes da Escola de Graduação em Ciências da Biosfera, Universidade de Hiroshima, Japão.

${ }^{3}$ Professora Associada C, Departamento de Química, Universidade Estadual de Londrina. E-mail: solci@uel.br 


\section{Introdução}

Os compostos carbonílicos (aldeídos e cetonas) desempenham um papel importante na química atmosférica como fontes de radicais livres e do aerossol orgânico e como precursores dos ácidos carboxílicos e espécies oxidantes (PIRES; CARVALHO, 1998). Formaldeído (FA) e acetaldeído (AA) são os mais abundantes, são diretamente emitidos para a atmosfera como também podem ser formados in-situ por meio de reações fotoquímicas que envolvem hidrocarbonetos provenientes de emissões biogênicas e antrópicas procedentes de fontes móveis e estacionárias.

Em áreas rurais limpas, FA e AA aparecem na atmosfera principalmente como produtos de reações de fotooxidação e ozonólise de hidrocarbonetos emitidos naturalmente (ROTTENBERG et al., 2004). Em ambientes urbanos, as fontes primárias de aldeídos incluem principalmente os processos de combustão; fontes secundárias importantes são os processos fotoquímicos de decomposição de hidrocarbonetos e outras espécies orgânicas presentes no ar. A queima incompleta de misturas oxigenadas em motores de combustão interna resulta no aumento das emissões primárias de FA e AA e conseqüentemente da formação de radicais livres, ozônio, ácidos carboxílicos e nitrato de peroxiacetila (GROSJEAN; MIGUEL; TAVARES, 1990; ANDRADE et al., 2002).

O conhecimento das relações entre os aldeídos e seus precursores é importante para se compreender os mecanismos de produção dos compostos orgânicos e do ozônio (O3) em atmosfera urbana (ARBILLA; OLIVEIRA, 1999).

As emissões de poluentes primários como óxidos de nitrogênio $\left(\mathrm{NO}_{\mathrm{x}}\right)$ decorrentes dos processos de combustão são fontes significativas de oxigênio atômico a partir da qual o ozônio pode formar-se. Os óxidos de enxôfre são também produzidos pela queima de combustíveis que contenham enxôfre em sua composição, além de serem gerados em processos biogênicos naturais, tanto no solo quanto na água (BRIMBLENCOMBE, 1996).

A principal via de desaparecimento dos FA e AA na atmosfera é através da destruição química causada por reações que ocorrem com radicais $\mathrm{OH}$, $\mathrm{OH}_{2}$ e $\mathrm{NO}_{3}$ e através da fotólise no caso do FA. (ANDRADE et al., 2002)

As concentrações dos aldeídos na atmosfera variam na ordem de poucos ppb em ar limpo $(0,2$ - 0,3 ppb em atmosfera marinha) chegando a altas concentrações em ambientes urbanos (> $140 \mathrm{ppb}$ ) (GROSJEAN, 1988; ZHOU; MOPPER, 1990; BAEZ et al., 2001).

Nas últimas décadas há em todo o mundo um crescente interesse nouso decombustíveis alternativos como o etanol, biodiesel e suas misturas oxigenadas produzidos por diferentes tipos de biomassa para minimizar a dependência do uso do petróleo, diminuir a emissão de $\mathrm{CO}, \mathrm{CO}_{2}$ e hidrocarbonetos e o impacto sobre o clima do planeta.

No Japão, o setor industrial consome cerca de $50 \%$ de energia sendo que mais de $78 \%$ utilizando combustíveis fósseis. Considerando o interesse do governo japonês em aumentar o uso de misturas oxigenadas como combustíveis, o objetivo deste estudo foi investigar a presença de aldeídos atmosféricos e sua relação com outros poluentes como ozônio, óxidos de nitrogênio e óxidos de enxôfre em Higashi-Hiroshima, Japão.

\section{Experimental}

\section{Local de amostragem}

As amostragens foram realizadas no Campus da Universidade de Hiroshima (Japão) em 13 e 14 de abril 19 e 20 de julho de 2005 localizado a 4 km sudoeste do centro urbano de Higashi-Hiroshima, a $35 \mathrm{~km}$ leste da cidade de Hiroshima, capital do estado, cuja população é de aproximadamente 115.000 habitantes (Figura 1). A população de Hiroshima é cerca de 1.114.000 habitantes sendo a cidade circundada por fábricas e indústrias. 


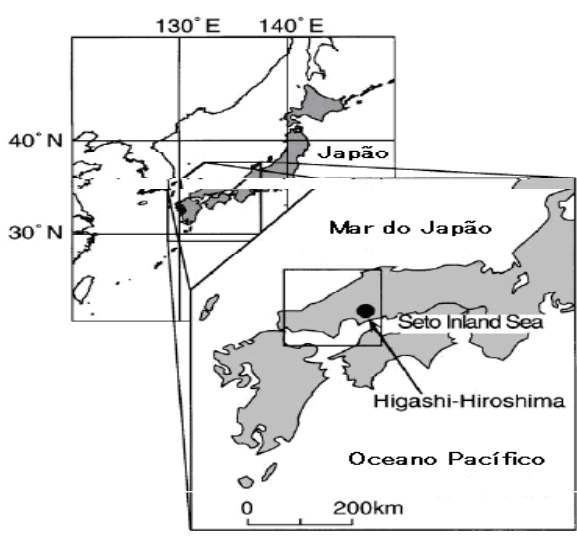

Figura 1. Localização do sítio de amostragem.

Coleta e determinação de formaldeído $e$ acetaldeído

Na primavera (13 e 14 de Abril/2005) e verão (19 e 20 de Julho/2005), amostras de ar ambiente foram coletadas durante $24 \mathrm{~h}$, fracionadas a cada $4 \mathrm{~h}$ sob vazão constante de $0,9 \mathrm{~L} \mathrm{~min}^{-1}$ utilizandose cartuchos de silica gel C18 impregnados com a solução 2,4-DNPH (Supelco, USA). Removedores de ozônio (Supelco, USA) foram utilizados para impedir possíveis interferências pela alta umidade noturna durante as amostragens (SIRJU; SHEPSON, 1995). Os cartuchos amostrados foram eluídos vagarosamente com $5 \mathrm{ml}$ de acetonitrila (Supelco, USA) e uma alíquota de $20 \mu \mathrm{l}$ foi injetada e analisada por cromatografia líquida de alta eficiência (JASCO PU-980) equipado com detector espectrofotométrico (JASCO 870-UV, Japão). As condições cromatográficas foram as seguintes: pré-coluna e coluna analítica Crestpack $\mathrm{C}_{18}$ (4.6 x $\left.250 \mathrm{~mm}\right)$; fase móvel isocrática água/

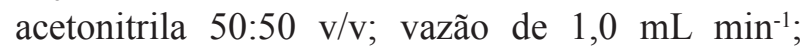
detector UV ajustado em $360 \mathrm{~nm}$. Formaldeído e acetaldeído foram identificados por comparação de seus tempos de retenção com os aldeídos padrões DNPH-hidrazonas (Tokyo Chemical Industry Co. Ltd.). Monitores para óxidos de nitrogênio ( $\mathrm{NO}$ e $\mathrm{NO}_{2}$ ), $\mathrm{SO}_{2}$ e $\mathrm{O}_{3}$ foram instalados no mesmo local de coleta e utilizando os seguintes equipamentos: $\mathrm{NO}_{x}$ (Thermo Environmental Instruments, Modelo-42S), $\mathrm{SO}_{2}$ (Thermo Environmental Instruments, Modelo
43C) e $\mathrm{O}_{3}$ (Oki-Electronics, Modelo OZM-G21). A radiação solar, tempertura e umidade relativa foram monitoradas através do sistema de aquisição de dados meteorológicos automático da Universidade de Hiroshima (HIRODAS).

\section{Resultados e discussão}

Todas as amostras foram coletadas sob boas condições meteorológicas, sem ocorrência de hidrometeoros. $\mathrm{Na}$ primavera a radiação solar variou de 0,002 a $0,855 \mathrm{kWm}^{-2}$, temperatura de 5,2 a $20,2{ }^{\circ} \mathrm{C}$ e umidade relativa de 20,5 a $99 \%$. No verão a radiação solar variou de 0,003 a $0,90 \mathrm{kWm}^{-2}$, temperatura de 23,2 a $33,4{ }^{\circ} \mathrm{C}$ e umidade relativa de 39 a $99 \%$.

A Tabela 1 apresenta a média e os intervalos de concentração do formaldeído e acetaldeído determinados na primavera (abril) e no verão (julho) de 2005 no Campus da Universidade de Hiroshima. $\mathrm{Na}$ primavera as concentrações de formaldeído variaram de 0,50 a $1,05(0,83 \pm 0,20) \mathrm{ppb}$ e do acetaldeído variaram de 0,17 a 1,31 $(0,46 \pm 0,43) \mathrm{ppb}$. No verão ( 19 e 20 de Julho de 2005) as concentrações do FA e AA variaram de 1,55 a 4,12 $(2,83 \pm 0,99) \mathrm{ppb}$ e 0,34 a $2,03(0,97 \pm 0,64) \mathrm{ppb}$, respectivamente.

A Figura 2 apresenta os perfis das concentrações dos aldeídos ao longo do período de amostragem na primavera e no verão.

$\mathrm{Na}$ primavera, observa-se a tendência dos aldeídos aumentarem no período diurno com acentuado decréscimo durante a noite. O FA aumentou em duas vezes no horário das 10 a 14 horas. A concentração do AA no mesmo período mostrou um aumento significativo de 8 vezes em relação à concentração noturna.

No verão, o perfil das concentrações dos aldeídos foi semelhante e os incrementos em suas concentrações em relação aos períodos diurnos e noturnos foram de 3 vezes para o FA e 6 vezes para o AA. 
A razão das concentrações FA\AA é usada para sugerir a origem dos aldeídos formados na atmosfera, sendo considerados os valores superiores a um (1) quando há predominância na formação de formaldeído via reações fotoquímicas (MONTEIRO et al., 2001). Foi encontrada a razão média FA/AA de 3,1 sugerindo que a emissão biogênica pode ter um impacto significante na concentração dos aldeídos no ar ambiente (VILLANUEVA-FIERRO; POPP; MARTINS, 2004).

Tabela 1 - Intervalo e média de concentração (ppb) dos aldeídos obtidos na primavera e verão de 2005 no Campus de Hirsohima, Japão.

\begin{tabular}{lcc}
\hline & FORMALDEÍDO & ACETALDEÍDO \\
PRIMAVERA & $0,50-1,05$ & $0,17-1,31$ \\
& $(0,83 \pm 0,20)$ & $(0,46 \pm 0,43)$ \\
& & \\
VERÃO & $1,55-4,12$ & $0,34-2,03$ \\
& $(2,83 \pm 1,00)$ & $(0,97 \pm 0,65)$ \\
\hline
\end{tabular}

As variações diurnas das concentrações dos aldeídos medidas no campus de Hiroshima apresentaram a mesma tendência comparada a estudos realizados por outros autores (ANDERSON et al., 1996; POSSANZINI et al., 1996; MONTEIRO et al., 2001; PINTO e SOLCI, 2007).

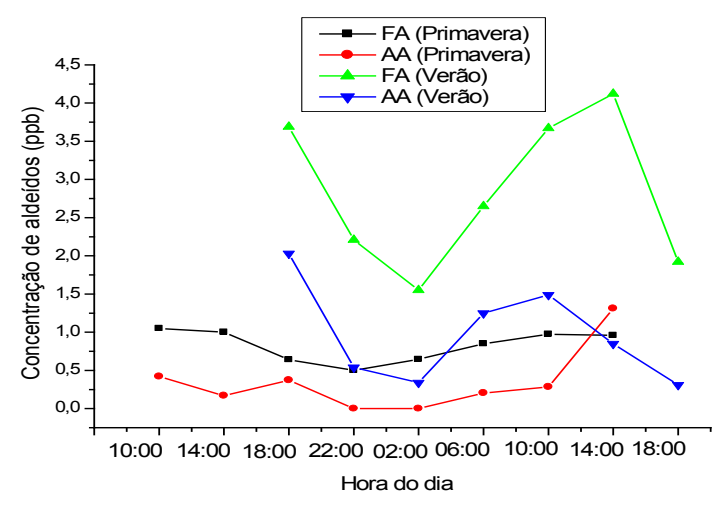

Figura 2. Perfis diários da concentração de formaldeído e acetaldeído durante a primavera e o verão de2005 no Japão.

\section{Primavera}

A Figura 3 apresenta as variações médias das concentrações de FA e AA, $\mathrm{O}_{3}, \mathrm{NO}$ e $\mathrm{NO}_{2}, \mathrm{SO}_{2}$ e radiação solar obtidas durante as amostragens realizadas na primavera. As concentrações de NO variaram de 0,01 a 11,4 ppb e de $\mathrm{NO}_{2}$ de 5,13 a $17,4 \mathrm{ppb}$. As concentrações de ozônio variaram no intervalo de 4,5 a 60 ppb sendo a maior concentração obtida no horário das 14 às 18 horas. Para o $\mathrm{SO}_{2}$ o intervalo de concentração foi de 0,6 a 3,2 ppb, característico de ambiente limpo.

Os perfis horários das concentrações de FA, AA, $\mathrm{NO}, \mathrm{NO}_{2}$ e $\mathrm{O}_{3}$ mostram, como esperado, a conversão de $\mathrm{NO}$ a $\mathrm{NO}_{2}$ e a formação fotoquímica de aldeídos e $\mathrm{O}_{3}$, uma vez que, quando ocorre o consumo de $\mathrm{NO}-\mathrm{NO}_{2}$ aumenta a concentração de FA, AA e $\mathrm{O}_{3}$.

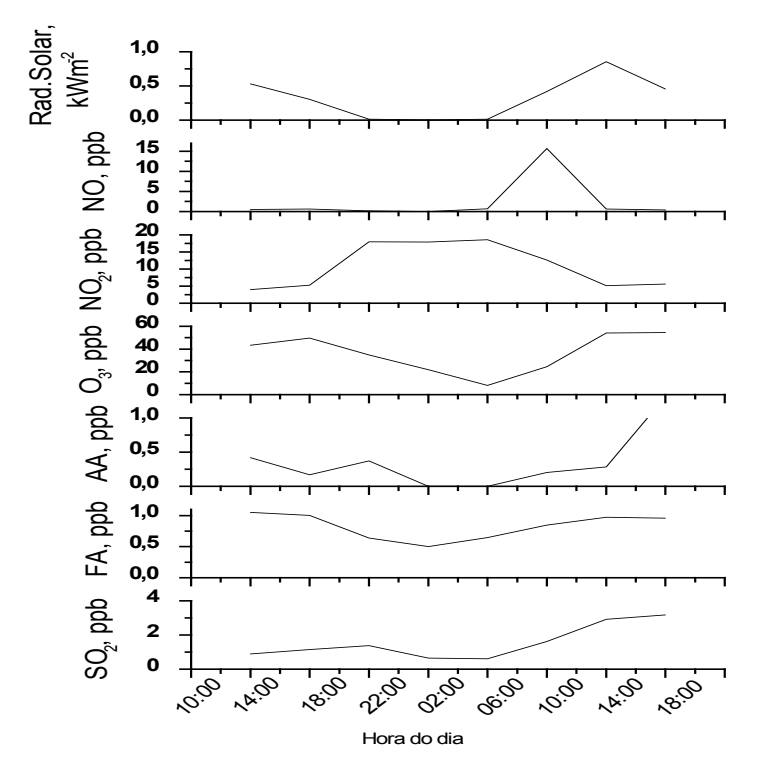

Figura 3. Perfil horário de $\mathrm{NO}, \mathrm{NO}_{2}, \mathrm{O}_{3}, \mathrm{SO}_{2}$, aldeídos e radiação solar durante a primavera (abril, 2005).

\section{Verão}

Os perfis das concentrações de $\mathrm{FA}, \mathrm{AA}, \mathrm{O}_{3}, \mathrm{NO}_{2}$, $\mathrm{SO}_{2}$ e radiação solar são apresentados na Figura 4. Nas primeiras horas do dia, as concentrações de FA e AA aumentam diminuindo ao entardecer quando a radiação solar é menos intensa indicando a 
formação dos aldeídos por processos fotoquímicos. A média da razão FA/AA encontrada foi de 3,72 sugerindo que as emissões biogênicas podem ter um impacto significante na concentração ambiente dos aldeídos.

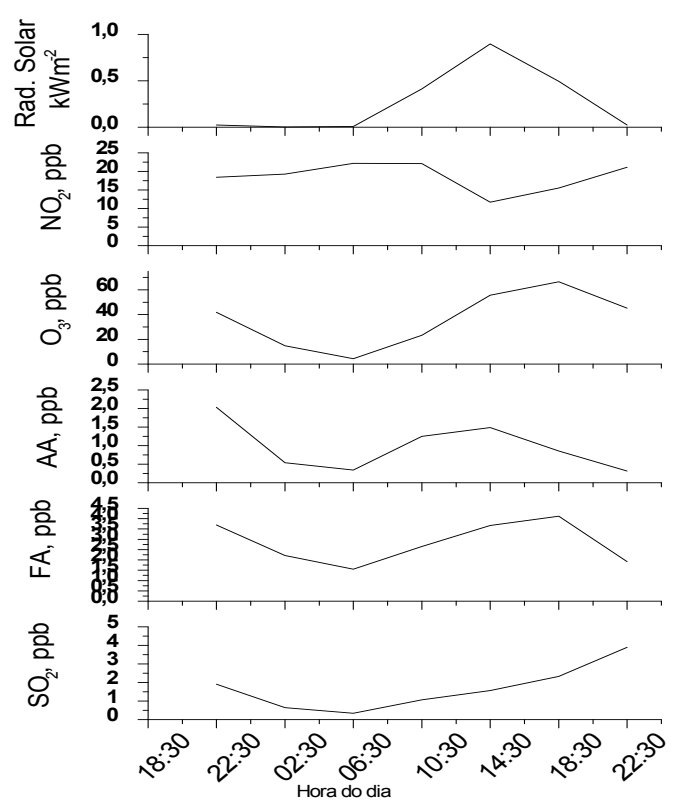

Figura 4. Perfil horário de $\mathrm{NO}, \mathrm{NO} 2, \mathrm{O} 3, \mathrm{SO} 2$, aldeídos e radiação solar durante o verão (julho, 2005). Obs. O NO não foi analisado por dificuldades técnicas.

Os FAeAAapresentaram uma grande semelhança nos perfis horários. No entanto, somente no verão o coeficiente de correlação obtido pela análise de regressão de FA x AA foi elevado $(r=0,73)$ indicando que estas espécies provavelmente provêm de fontes e processos similares de formação. À tarde grande parte dos aldeídos tendem a desaparecer devido aos processos de decomposição fotoquímica. A decomposição fotoquímica de aldeídos e ozônio induzida pela luz solar leva a um aumento no número de radicais livres no ar (FINLAYSONPITTS; PITTS, 2002).

Mesmo que a intensidade de radiação solar no verão tenha sofrido pequena diferença comparada com a primavera, as concentrações de FA e AA no verão foram maiores acompanhando as altas concentrações das espécies precursoras $\left(\mathrm{NO}_{2}\right.$ e $\left.\mathrm{O}_{3}\right)$ e evidenciando a formação dos aldeídos através de processos fotoquímicos.

$\mathrm{SO}_{2}$ é um poluente comum no ar emitido diretamente para a atmosfera principalmente por fontes de origem antrópica como a combustão de combustíveis fósseis contendo enxôfre (GUPTA et al., 2003). As concentrações de $\mathrm{SO}_{2}$ na primavera e no verão não ultrapassaram 4 ppb em consonância com os valores encontrados em 2006 no mesmo local (THAM; TAKEDA; SAKUGAWA, 2008). Como outras fontes de combustão (gás natural, carvão, madeira) não são significantes em Higashi Hiroshima, provavelmente o $\mathrm{SO}_{2}$ foi primariamente emitido por veículos movidos a diesel.

$\mathrm{NO}_{2}$ é um poluente importante relacionado ao tráfego veicular e precursor da formação fotoquímica do smog e do ozônio em baixa atmosfera (FINLAYSON-PITTS; PITTS, 2002).

$\mathrm{O} \mathrm{NO}_{2}$ é principalmente formado através da oxidação do NO (HAN; NAEHAR, 2006) e as concentrações de $\mathrm{NO}_{2}$ encontradas no período de amostragem foram similares às obtidas por Tham, Takeda e Sakugawa (2008).

O ozônio é um poluente do ar muito reativo e tem um papel muito importante nas reações fotoquímicas que ocorrem na troposfera. Tem como importantes precursores os óxidos de nitrogênio $\left(\mathrm{NO}_{\mathrm{x}}\right)$ e os compostos orgânicos voláteis (COVs) que participam do complexo processo fotoquimico na presença de luz solar e calor (DERWENT et al., 2003; RIBAS; PENUELAS, 2004). O perfil de concentração do $\mathrm{O}_{3}$ comparado aos dados de 2006 foi semelhante. Observa-se ainda que a formação do $\mathrm{O}_{3}$ no verão foi incrementada devido ao aumento de temperatura uma vez que a radiação solar na primavera e verão foi similar. (PUDASAINEE et al., 2006).

A Tabela 4 apresenta resultados obtidos em diferentes locais de amostragem (urbano, suburbano e rural) onde as concentrações dos aldeídos foram medidas. São apresentadas ainda as razões FA/AA. É possível observar que em locais rurais limpos com 
menor influência de emissões antrópicas, as razões FA/AA são superiores a 2. Em ambientes urbanos e semi-urbanos, exceto no Brasil, FA/AA são superiores a um. No Brasil, a razão FA/AA tende a valores inferiores a $1 \mathrm{em}$ atmosfera impactada por emissões veiculares devido ao uso de combustíveis oxigenados.

É importante salientar que em áreas limpas, a razão FA/AA é maior refletindo a participação de hidrocarbonetos reativos de origem biogênica e a prevalência de formação fotoquímica de formaldeído (PINTO; SOLCI, 2007).

Tabela 4 - Concentração dos aldeídos (ppb) e FA/ AA medidos em diferentes localizações e períodos.

\begin{tabular}{|c|c|c|c|c|c|}
\hline Local & FA & $\mathbf{A A}$ & & ríodo & Referência \\
\hline \multicolumn{6}{|l|}{ Urbano } \\
\hline $\begin{array}{l}\text { Los Angeles } \\
\text { (EUA) }\end{array}$ & 11 - 39 & $13-24$ & 1,85 & 1980 & $\begin{array}{c}\text { Grosjean. } \\
(1988)\end{array}$ \\
\hline $\begin{array}{l}\text { Londrina } \\
\text { (Brasil) }\end{array}$ & $0,24-9,46$ & $2,81-10,83$ & 0,86 & $\begin{array}{l}\text { Inverno } \\
2002\end{array}$ & $\begin{array}{c}\text { Pinto e Solci } \\
\text { (2007) }\end{array}$ \\
\hline $\begin{array}{l}\text { Roma } \\
\text { (Itália) }\end{array}$ & $8,2-17$ & $2,9-6,6$ & 2,65 & $\begin{array}{l}\text { Verão } \\
1995\end{array}$ & $\begin{array}{c}\text { Possanzini et al. } \\
\text { (1996) }\end{array}$ \\
\hline $\begin{array}{l}\text { Osaka } \\
\text { (Japão) }\end{array}$ & $0,1-4,3$ & $0,6-3,5$ & 1,26 & $\begin{array}{l}\text { Inverno } \\
1997\end{array}$ & $\begin{array}{l}\text { Nguyen et al. } \\
\text { (2001) }\end{array}$ \\
\hline $\begin{array}{l}\text { São Paulo } \\
\text { (Brasil) }\end{array}$ & $1,4-9,7$ & $1,0-10,2$ & 0,92 & $\begin{array}{l}\text { Verão } \\
1998\end{array}$ & $\begin{array}{l}\text { Nguyen et al. } \\
\text { (2001) }\end{array}$ \\
\hline $\begin{array}{l}\text { São Paulo } \\
\text { (Brasil) }\end{array}$ & $1,05-46,7$ & $1,2156,6$ & 0,82 & $\begin{array}{l}\text { Inverno } \\
1999\end{array}$ & $\begin{array}{l}\text { Monteiro et al. } \\
\quad(2001)\end{array}$ \\
\hline \multicolumn{6}{|l|}{ Sub-urbano } \\
\hline $\begin{array}{l}\text { Hiroshima } \\
\text { (Japão) }\end{array}$ & $0,50-1,05$ & $13-24$ & 1,80 & $\begin{array}{l}\text { Primavera } \\
2005\end{array}$ & $\begin{array}{l}\text { Grosjean. } \\
\text { (1988) }\end{array}$ \\
\hline $\begin{array}{l}\text { Hiroshima } \\
\text { (Japão) }\end{array}$ & $1,55-4,12$ & $2,81-10,83$ & 2,81 & $\begin{array}{l}\text { Verão } \\
2005\end{array}$ & $\begin{array}{c}\text { Pinto e } \\
\text { Solci(2007) }\end{array}$ \\
\hline $\begin{array}{l}\text { Londrina } \\
\text { (Brasil) }\end{array}$ & $0,62-1,88$ & $2,9-6,6$ & 0,63 & $\begin{array}{l}\text { Outono } \\
2001\end{array}$ & $\begin{array}{l}\text { Possanzini } \\
\text { et al.(1996) }\end{array}$ \\
\hline $\begin{array}{l}\text { Lille Vally } \\
\text { (Dinamarca) }\end{array}$ & $0,3-1,8$ & $0,6-3,5$ & 1,61 & $\begin{array}{l}\text { Primavera } \\
1994\end{array}$ & $\begin{array}{l}\text { Nguyen et } \\
\text { al. (2001) }\end{array}$ \\
\hline \multicolumn{6}{|l|}{ Rural } \\
\hline $\begin{array}{l}\text { Londrina } \\
\text { (Brasil) }\end{array}$ & $0,64-1,41$ & $0,10-0,87$ & 2,29 & $\begin{array}{l}\text { Inverno } \\
2002\end{array}$ & $\begin{array}{l}\text { Pinto e Solci } \\
\quad(2007)\end{array}$ \\
\hline $\begin{array}{l}\text { Ontário } \\
\text { (Canadá) }\end{array}$ & 1,7 & 0,52 & 3,27 & $\begin{array}{l}\text { Verão } \\
1988\end{array}$ & $\begin{array}{l}\text { Shepson et } \\
\text { al. (1991) }\end{array}$ \\
\hline $\begin{array}{l}\text { Alpes } \\
\text { (Alemanha) }\end{array}$ & $0,5-3,2$ & $0,3-1,2$ & 2,46 & $\begin{array}{l}\text { Outono } \\
1991\end{array}$ & $\begin{array}{l}\text { Slemr et al. } \\
\text { (1996) }\end{array}$ \\
\hline
\end{tabular}

\section{Conclusões}

As concentrações de FA e AA encontradas na Universidade de Hiroshima em abril e julho de 2005 apresentaram perfis similares quanto ao comportamento temporal em concordância com outros estudos. Os níveis dos aldeídos durante o dia foram superiores ao período noturno sendo sugerida a atividade fotoquímica como a principal fonte destes aldeídos. Parâmetros meteorológicos, $\mathrm{O}_{3}$, $\mathrm{NO}, \mathrm{NO}_{2}$ e $\mathrm{SO}_{2}$ comparados aos aldeídos indicaram a formação secundária dos FA e AA, tendo como possíveis fontes as emissões de origem antrópica e biogênica. Comparações das razões FA/AA medidas neste estudo com estudos anteriores sugerem uma forte contribuição de fontes biogênicas na formação destes aldeídos.

\section{Agradecimentos}

CAPES e CNPq. JAICA pela bolsa de estudos concedida a Sonia N. Nomi.

\section{Referências}

ANDERSON, L. G.; LANNING, J. A.; BARRELL, R.; MIYAGISHIMA, J.; JONES, R. H.; WOLFE, P. Sources and sinks of formaldehyde and acetaldehyde: an analysis of denvers ambient concentration data. Atmospheric Environment, Oxford, v. 30, n. 12, p. 2113-2123, 1996.

ANDRADE, M. V. A.; PINHEIRO, H. L. C.; PEREIRA, A. F. P.; ANDRADE, J. B. Compostos carbonílicos atmosféricos: fontes, reatividade, níveis de concentração e efeitos toxicológicos. Química Nova, São Paulo, v. 25, n. 6, p. 1117-1131, 2002.

ARBILlA, G.; OLIVEIRA, K. M. P. G. Otimização de um mecanismo fotoquímico para a simulação da atmosfera urbana brasileira. Química Nova, São Paulo, v. 22, n. 6, p. 790-800, 1999.

BAEZ, A. P.; PADILLA, H.; CERVANTES, J.; PEREYRA, D.; TORRES, M. C.; GARCIA, R.; BELMONT, R. Preliminary study of the determinations of ambient carbonyls in Xalapa City. Atmospheric Environment, Oxford, v. 35, n. 10, p. 1813-1819, 2001.

BRIMBLENCOMBE, P. Air composition \& chemistry. Cambridge: Cambridge University Press, 1996. 
DERWENT, R. G.; JENKIN, M. E.; SAUNDERS, S. M.; PILLING, M. J.; SIMMONDS, P. G.; PASSANT, N. R. Phtochemical ozone formation in North West Europe and its control. Atmospheric Environment, Oxford, v. 37, n. 14, p. 1983-1991, 2003.

FINLAYSON-PITTS, B. J.; PITTS, J. N. Chemistry of the upper and lower atmosphere. Washington: Academic Press, 2002.

GROSJEAN, D. Formaldehyde and other carbonyls in Los Angeles air. Environmental Science and Technolology, Washington, v. 16, n. 5, p. 254- 262, 1988.

GROSJEAN, D.; MIGUEL, A. H.; TAVARES, T. M. Urbana air pollution in Brazil: acetaldehyde and other carbonyls. Atmospheric Environment, Oxford, v. 24, n. 1, p. 101-106, 1990.

GUPTA, A.; KUMAR, R.; KUMARI, K. M.; SRIVASTAVA, S. S. Measurement of NO2, HNO3, NH3 and $\mathrm{SO} 2$ and related particulate matter at a rural site in Rampur, India. Atmospheric Environment, Oxford, v. 37, n. 34, p. 4837-4846, 2003.

HAN, X.; NAEHAR, L. P. A review of traffic-related air pollution exposure assessment studies in the developing world. Environment International, Amsterdam, v. 32, n. 1, p.106-120, 2006.

MONTEIRO, L.; VASCONCELLOS, P. C.; SOUZA, S. R.; PIRES, M. A.; SANCHEZ-CCOYLLO, O. R.; ANDRADE, M. F.; CARVALHO, L. R. Measurements of atmospheric carboxylic acids and carbonyl compounds in São Paulo city, Brazil. Environmental Science and Technology, Washington, v. 35, n. 15, p. 3071-3081, 2001.

PINTO, J. P.; SOLCI, M. C. Comparison of rural and urban atmospheric aldehydes in Londrina, Brazil. Journal of Brazilian Chemical Society, São Paulo, v. 18, n. 5, p. 928-936, 2007.

PIRES, M.; CARVALHO, L. R. F. Na artifact in air carbonyls sampling using C18 DNPH-coated cartridge. Anaytica Chimica Acta, Amsterdam, v. 367, n. 1, p. 223231, 1998.

POSSANZINI, M.; DI PALO, V.; PETRICCA, M.; FRATARCANGELI, R.; BROCCO, D. Measuremnts of lower carbonyls in Rome ambient air. Atmospheric Environment, Oxford, v. 30, n. 22, p. 3757-3764, 1996.
PUDASAINEE, D.; SAPKOTA, B.; SHRESTHA, M. L.; KAGA, A.; KONDO, A.; INOUE, Y. Ground level ozone concentrations and its association with $\mathrm{NOx}$ and materological parameters in Kathmandu valley, Nepal. Chemosphere, Oxford, v. 15, p.675-685, 2006.

RIBAS, A.; PENUELAS, J. Temporal patterns of surface ozone levels in different habitats of the North Western Mediterranean basin. Atmospheric Environment, Oxford, v. 38, n. 7, p. 985-992, 2004.

ROTTENBERG, S.; KUHN, U.; SCHEBESKE, G.; OLIVA, S. T.; TAVARES, T. M.; KESSELMEIER, J. Exchange of short-chain aldehydes between Amazonian vegetation and the atmosphere. Ecological applications, Washington, v. 14, supplement, p. 247-267, 2004

SIRJU, A. P.; SHEPSON, P. B. Laboratory and field investigation of the DNPH cartridge technique for the atmospheric carbonyl compounds. Environmental Science and Technology, Washington, v. 29, n. 2, p. 384392, 1995.

THAM, Y. W. F.; TAKEDA, K.; SAKUGAWA, H. Exploring the correlation of particulate PAHs, sulfur dioxide, nitrogen dioxide and ozone, a preliminary study. Water Air Soil Pollution, Dordrecht, v. 194, n. 1/4, p. 5-12, 2008.

VILLANUEVA-FIERRO, I.; POPP, C. J.; MARTINS, R. S. Biogenic emissions and ambient concentrations of hydrocarbons, carbonyl compounds and organic acids from ponderosa pine and cottonwood trees at rural and forested sites in central New Mexico. Atmospheric Environment, Oxford, v. 38, n. 2, p. 249-260, 2004.

ZHOU, X.; MOPPER, K. Measurements of sub-partsper-billion levels of carbonyl compounds in marine air by a simple cartridge trapping procedure followed by liquid chromatography. Environmental Science and Technolog, Washington, v. 24, n. 10, p. 1482-1485, 1990.

Recebido em 25 Setembro, 2009 - Received on September 25, 2009. Aceito em 12 Abril, 2010 - Accepted on April 12, 2010. 